\title{
Analyzing voting behaviour in the United States banking sector through eigenvalue decomposition
}

\section{Juan Pineiro-Chousa, Marcos Vizcaíno-González and Jérôme Caby}

Using data about votes emitted by funds in corporate meetings held by United States banks from 2003 to 2013, we build a correlation matrix of the voting pattern observed in the banking industry in order to compute its eigenvalue decomposition. Our results indicate that there is a main underlying feature that contributes to explain this voting behaviour. Also, a dimensionality reduction of the correlation matrix could be accomplished, so that a subset of the original matrix can replicate the data sample. These findings suggest that there may be a sort of homogeneous or systematic component when it comes to explain the voting pattern into the banking industry.

Keywords: voting behaviour, eigenvalue decomposition, singular value decomposition, spectral decomposition.

JEL codes: G01, G21, G32, G34 


\section{Introduction}

Understanding the voting pattern observed in corporate meetings is one of the major topics into the corporate governance field. The aim of this research is to address this open issue, investigating the suitability of an eigenvalue decomposition approach for this purpose. Using data on votes emitted by funds in corporate meetings organized by United States banks from 2003 to 2013, we build a correlation matrix that collects the voting pattern of the industry. Computing the eigenvalue decomposition for this correlation matrix, we identify the underlying factors that describe the observed voting behaviour.

\section{Financial applications of eigenvalue decomposition}

Academic research offers a wide set of remarkable results regarding applications of key methodologies from random matrix theory to financial markets and business management. In fact, random matrix theory provides useful techniques in order to discover the statistical composition of correlation matrices when it comes to investigate oscillations in market prices of stocks or other financial assets (Laloux et al., 2000; Plerou et al., 2000; Laloux et al., 1999; Plerou et al., 1999). The same approach has been proposed in order to research the volatility (Kulkarni and Deo, 2007) and intraday oscillations (Rak et al., 2006) of a stock market, or to address the study of correlations in financial series (Burda and Jurkiewicz, 2004) and emerging markets (Pan and Sinha, 2007; Wilcox and Gebbie, 2007). In a more general sense, the random matrix theory framework has been suggested as a suitable schema in order to analyze the correlated performance between distinct companies (Rosenow et al., 2000), or to investigate the 
correlation between business cycles of different countries from the European Union (Ormerod, 2005).

The researches in this area frequently rely on the assumption that financial markets can be understood as particular cases of a complex system, so that significant patterns can be identified as different market states reflecting the occurrence of anomalies as financial crises by means of the computation of eigenvalues and eigenvectors of a correlation matrix (Sandoval and Franca, 2012; Münnix et al., 2011). In particular, the largest eigenvalue of a correlation matrix has been referred to capture the maximum quantity of the variance that can be explained by a unique underlying feature (Friedman and Weisberg, 1981). That is why computing the associated eigenvector to this largest eigenvalue allows splitting a set of stocks into different subsets, with useful applications for portfolio optimization (Gopikrishnan et al., 2001). In addition to the importance granted to the largest eigenvalue, it has been stated that the second and third largest eigenvalues should also be considered, since they are likely to supply key information regarding the risk incorporated into the stock data (Namaki et al., 2011).

\section{Data}

The data used in this research comprise votes disclosed by the largest large cap funds and by socially responsible investing funds in corporate meetings organized by United States banks. These votes concern managerial proposals about executive compensation and directors' election, and they are collected by the non-profit and non-partisan organization ProxyDemocracy, which has been lately considered an adequate supplier of voting data for research purposes (Burns and Minnick, 2013). The final sample includes 95234 votes for 309 banks regarding the period from 2003 to 2013. 
We compute the ratio $\frac{1+\text { pro }}{1+\text { non-pro }}-1$, where pro represents the proportion of pro-votes emitted for a certain bank and year, and non-pro stands for the proportion of non-pro votes, aggregating abstains and against votes (Gregory-Smith \& Main, 2013), for the same bank and year. This ratio informs about the level of support to managerial proposals, considering that the value is negative when the non-pro votes dominate, and the value is positive otherwise. We also compute the ratio $\ln \left(\frac{\text { votes }}{\text { funds }}\right)$, where votes is the total number of votes emitted for a certain bank and year, and funds is the total number of funds emitting votes for the same bank and year. Thus, this ratio measures the average number of votes emitted by fund. Finally, we multiply this average plus the level of support in order to obtain an indicator of the voting pattern. Once the voting pattern is calculated for each of the 309 banks and for each of the 11 years in our sample, we compute the pairwise correlation coefficients in order to build a correlation matrix for the voting pattern into the whole United States banking sector. As a result, we obtain a 309-row symmetric square matrix to be used as the input for our calculations.

\section{Results}

Given that our correlation matrix is a normal matrix, its singular values match the eigenvalues, and its singular vectors match its eigenvectors. So, we accomplish the singular value decomposition of the matrix in order to conquer the eigenvalues and the eigenvectors. As a result, we obtain 309 eigenvalues ordered from higher to lower. In Table 1 we report the 25 largest eigenvalues:

[Insert Table 1 about here] 
In addition, we supply a graph representing the 50 largest eigenvalues:

[Insert Fig. 1 about here]

From the data reported, it can be pointed out that the first eigenvalue clearly outstands above all the other ones, showing a value that is around $40 \%$ higher than the second eigenvalue. That is to say that the largest eigenvalue explains a great part of the variance in this sample of data (Friedman and Weisberg, 1981), leading us to conclude that there is a main underlying factor that significantly contributes to explicate the voting pattern observed in the whole United States banking sector.

Once the existence of a dominant underlying factor has been established, we turn our sights towards the eigenvector associated to this largest eigenvalue in order to consider the possibility that a certain subset of the sample data can be defined (Gopikrishnan et al., 2001). We observe that 68 of the 309 values contained in this eigenvector are null, representing a $22 \%$ of the total. This finding suggests that a significant dimensionality reduction of the correlation matrix can be easily made, that is, the voting behaviour observed in the United States banking sector can be satisfactorily explained by a subset of the companies that constitute the whole industry. This result can be considered a signal of the voting behaviour performing homogeneously or systematically to a certain degree along the banking sector.

In order to further explore these findings, we also consider the importance of the second and the third largest eigenvalues showed in Table 1 (Namaki et al., 2011). When it comes to analyze the eigenvectors associated to these two eigenvalues, the conclusions are exactly the same than the one achieved previously. For the second and third eigenvalues, we discover that 68 of the 309 values contained in their respective eigenvectors are null, confirming the former analysis and reinforcing our conclusions. 
[Insert Table 2 about here]

\section{Conclusions}

Using data about the votes emitted by funds in corporate meetings held by United States banks, we build a correlation matrix that captures the voting pattern of the industry in order to compute its eigenvalue decomposition. Our results show that the largest eigenvalue clearly outstands above all the other ones, indicating that there is a main explanatory factor for the voting pattern and suggesting that there may be a systematic or homogenous voting component in the whole United States banking industry. In addition, we explore the eigenvector associated to this largest eigenvalue, finding that $22 \%$ of the values are null, indicating that a dimensionality reduction of the correlation matrix could be accomplished, so that a subset of the original sample can be used to replicate the whole dataset, reinforcing the previous conclusion. We repeat the analysis with the second and third largest eigenvalues, demonstrating the consistency of our results. 


\section{References}

Burda, Z. and Jurkiewicz, J. (2004) Signal and noise in financial correlation matrices, Physica A: Statistical Mechanics and its Applications, 344, 67-72.

Burns, N. and Minnick, K. (2013) Does Say on Pay Matter? Evidence from Say on Pay Proposals in the United States, Financial Review, 48, 233-58.

Friedman, S. and Weisberg, H. F. (1981) Interpreting the first eigenvalue of a correlation matrix, Educational and Psychological Measurement, 41, 11-21.

Gopikrishnan, P., Rosenow, B., Plerou, V. and Stanley, H. E. (2001) Quantifying and interpreting collective behavior in financial markets, Physical Review E, 64, 035106.

Kulkarni, V. and Deo, N. (2007) Correlation and volatility in an Indian stock market: A random matrix approach, The European Physical Journal B-Condensed Matter and Complex Systems, 60, 101-9.

Laloux, L., Cizeau, P., Bouchaud, J. and Potters, M. (1999) Noise dressing of financial correlation matrices, Physical Review Letters, 83, 1467-70.

Laloux, L., Cizeau, P., Potters, M. and Bouchaud, J. (2000) Random matrix theory and financial correlations, International Journal of Theoretical and Applied Finance, 3, $391-7$.

Münnix, M., Shimada, T., Schäfer, R., Leyvraz, F., Seligman, T., Guhr, T. and Stanley, H. (2011) Identifying states of a financial market. Scientific reports, 2, 644-. 
Namaki, A., Shirazi, A., Raei, R. and Jafari, G. (2011) Network analysis of a financial market based on genuine correlation and threshold method, Physica A: Statistical Mechanics and its Applications, 390, 3835-41.

Ormerod, P. (2005) The Convergence of European Business Cycles 1980--2004, Acta Physica Polonica B, 36, 2747-56.

Pan, R. K. and Sinha, S. (2007) Collective behavior of stock price movements in an emerging market, Physical Review E, 76, 46116-24.

Plerou, V., Gopikrishnan, P., Rosenow, B., Amaral, L. N. and Stanley, H. (2000) A random matrix theory approach to financial cross-correlations, Physica A: Statistical Mechanics and its Applications, 287, 374-82.

Plerou, V., Gopikrishnan, P., Rosenow, B., Amaral, L. A. N. and Stanley, H. E. (1999) Universal and nonuniversal properties of cross correlations in financial time series, Physical Review Letters, 83, 1471-4.

Rak, R., Kwapień, J. and Oświęcimka, P. (2006) Correlation matrix decomposition of WIG20 intraday fluctuations, Acta Physica Polonica B, 37, 3123-32.

Rosenow, B., Plerou, V., Gopikrishnan, P., Amaral, L. A. N. and Stanley, H. E. (2000) Application of random matrix theory to study cross-correlations of stock prices, International Journal of Theoretical and Applied Finance, 3, 399-403.

Sandoval, L. and Franca, I. D. P. (2012) Correlation of financial markets in times of crisis, Physica A: Statistical Mechanics and its Applications, 391, 187-208. 
Wilcox, D. and Gebbie, T. (2007) An analysis of cross-correlations in an emerging market, Physica A: Statistical Mechanics and its Applications, 375, 584-98. 